\title{
Statistical Measurement of Polar Displacements in Complex Oxides
}

\author{
Leixin Miao, Debangshu Mukherjee, Greg Stone and Nasim Alem
}

Department of Materials Science and Engineering, The Pennsylvania State University, University Park, PA, USA

Ferroelectrics are crystals with a spontaneous, switchable electrical polarization [1]. According to the modern theory of ferroelectricity, the polarization is a function of the polar displacement and the Born effective charge [2]. In complex oxides this is defined as second-order Jahn-Teller displacements in proper ferroelectrics, where the primary order parameter is the $B$ site displacement with respect to the oxygen octahedral cage in $\mathrm{ABO}_{3}$ perovskites [3]. In improper ferroelectrics, this is a first order Jahn-Teller distortion, where the rotation of the oxygen octahedral cages results in the absence of inversion symmetry [4]. SubÅngström resolution imaging with aberration correction has allowed the direct measurement of atom positions with scanning transmission electron microscopy [5]. Aberration corrected scanning transmission electron microscopy (STEM) has been used to image the evolution of domain walls in $\mathrm{BiFeO}_{3}$ thin films, and have been used to image the presence of novel phenomena like polarization vortices in oxide superlattices $[6,7]$.

Scanning distortions like jitter and non-linear scan drift in STEM can cause the local displacement measurement prone to error [8]. To overcome these challenges we use a relative statistical measurement to quantify sub $10 \mathrm{pm}$ displacements in complex oxides. An average summation of atom positions generates a measurement with reduced random noise and jitter. Secondly, a relative measurement between the neighboring atomic species minimizes the contribution of non-linear drift. Finally, a statistical sampling leads to significantly higher accuracy in the displacement measurement, with the accuracy being inversely proportional to the square root of the number of sampling points according to the central limit theorem [9].

Figure 1a shows the annular dark field STEM image of $\mathrm{CaFeO}_{2.5}-\mathrm{SrFeO}_{2.5}$ thin films grown on a $\mathrm{SrTiO}_{3}$ substrate. Density functional theory predicts three structures with equal energy, distinguished by the orientation of Fe tetrahedral layers [10]. Statistical measurement of the horizontal displacements of the Bsites which are the tetrahedral and octahedral Fe layers in film and the Ti atoms in the substrate is shown in Figure 1b. This shows no horizontal displacements in the substrate, and chiral tetrahedral displacements in the film. The overlaid predicted displacements from density functional theory demonstrate strong agreement between the statistically sampled experimental measurements and theoretical predictions. Through properly summing the unit cell displacements, we also demonstrate the horizontal displacements maintaining their chirality, while the smaller magnitude demonstrates the effect of the substrate on the polar displacement. Another example pertains to probing the atomic structure in $\mathrm{LiNbO}_{3}$ across a $180^{\circ}$ domain wall (Fig. 1c). Through summing the unit cells and quantifying the average displacements in $\mathrm{LiNbO}_{3}$, statistical measurements parallel and perpendicular to the domain wall indicate that Ising nature of the domain wall with $30 \mathrm{pm}$ relative displacement of $\mathrm{NbO}_{6}$ octahedra across the wall. Additionally, the $\mathrm{NbO}_{6}$ octahedra also indicate displacements on the order of $10 \mathrm{pm}$ perpendicular to the wall, demonstrating the presence of a Néel wall. This research will present our statistical metrology approach allowing quantification of the displacements beyond the resolution limit of the microscope [11]. 
References:

[1] M.E. Lines and A.M. Glass, Principles and Applications of Ferroelectrics and Related Materials. Oxford University Press, 1 edition, 1977.

[2] R. Raffaele, Ferroelectrics 151(1) (1994), p. 49.

[3] P. S. Halasyamani and K. R. Poeppelmeier, Chemistry of Materials 10(10) (1998), p. 2753.

[4] N.A. Benedek and C. J. Fennie, Phys. Rev. Lett. 106 (2011), p. 107204.

[5] P.D. Nellist et al, Science 305(5691) (2004), p. 1741.

[6] C.T. Nelson et al, Nano Letters 11(2) (2011), p. 828.

[7] A.K. Yadav et al, Nature 530 (2016), p. 198.

[8] L. Jones and P. D. Nellist, Microscopy and Microanalysis 19(4) (2013), p. 1050.

[9] J.L. Doob, Stochastic processes, 1953.

[10] J. Young et al, Journal of the American Chemical Society, 2017.

[11] Leixin Miao and Debangshu Mukherjee contributed equally to the work. This work was funded by the Penn State MRSEC, Center for Nanoscale Science, under the award NSF DMR-1420620.

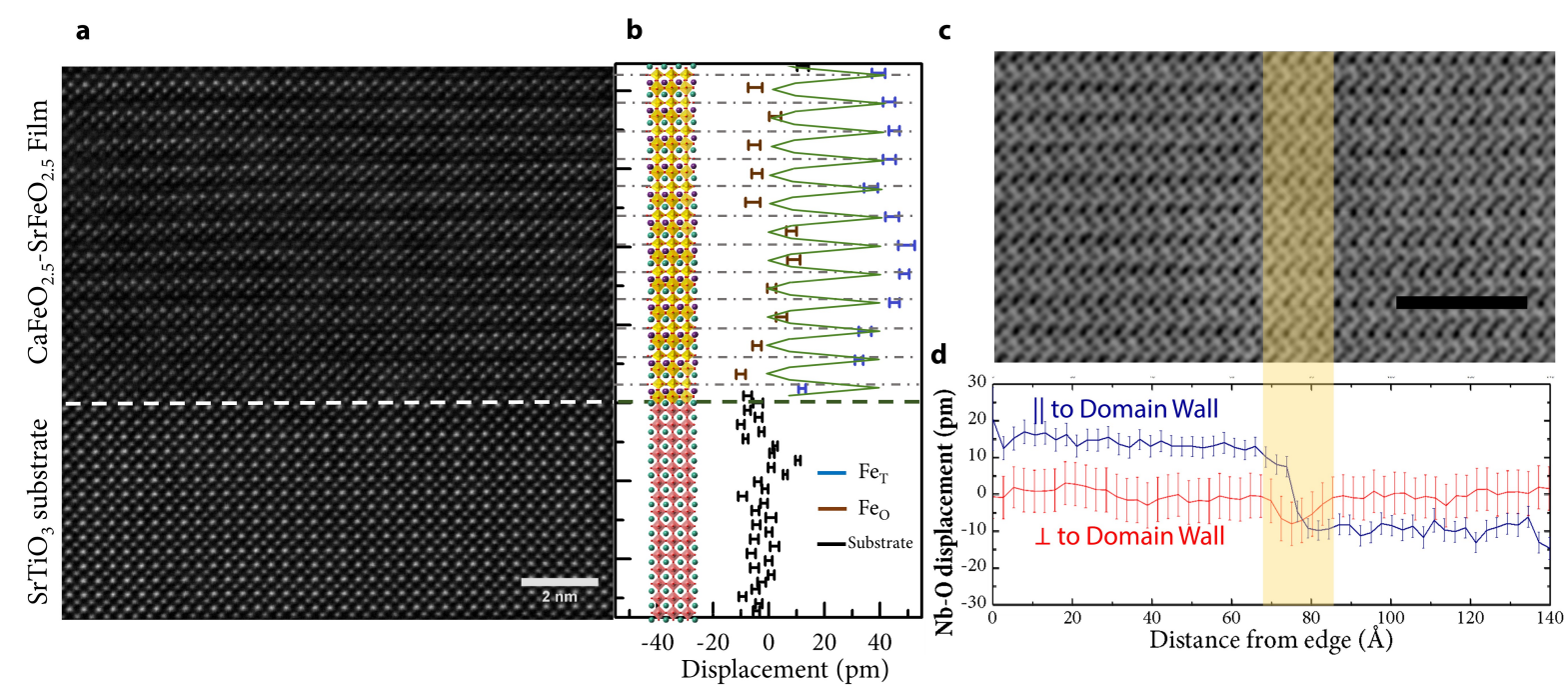

Figure 1. (a), ADF-STEM image of $\mathrm{CaFeO}_{2.5}-\mathrm{SrFeO}_{2.5}$ film grown on a $\mathrm{SrTiO}_{3}$ substrate. (b) Statistical measurement of horizontal displacements Fe displacements with respect to $\mathrm{Ca}$ and $\mathrm{Sr}$ positions, compared with DFT predictions (in green). (c) ABF-STEM image of $\mathrm{LiNbO}_{3}$ showing $\mathrm{Nb}$ and $\mathrm{O}$ atoms. The scale bar is $2 \mathrm{~nm}$. (d) Statistical measurements of $\mathrm{NbO}_{6}$ displacements I and $\perp$ to the domain wall demonstrating a mixed domain wall. 